\title{
Tracking System with Re-identification Using a RGB String Kernel
}

\author{
Amal Mahboubi ${ }^{1}$, Luc Brun ${ }^{1}$, \\ Donatello Conte ${ }^{2}$, Pasquale Foggia ${ }^{3}$, and Mario Vento ${ }^{3}$ \\ 1 GREYC UMR CNRS 6072, Equipe Image ENSICAEN \\ 6, boulevard Maréchal Juin F-14050 Caen, France \\ amal.mahboubi@unicaen.fr, luc.brun@ensicaen.fr \\ 2 Université François-Rabelais de Tours, LI EA 6300 \\ 64, Avenue Jean Portalis, F-37200, Tours, France \\ donatello.conte@univ-tours.fr \\ 3 Dipartimento di Ingegneria dell'Informazione, \\ Ingegneria Elettrica e Matematica Applicata \\ Università di Salerno, Via Ponte Don Melillo, 1 I-84084 Fisciano (SA), Italy \\ \{pfoggia,mvento\}@unisa.it
}

\begin{abstract}
People re-identification consists in identifying a person that comes back in a scene where it has been previously detected. This key problem in visual surveillance applications may concern single or multi camera systems. Features encoding each person should be rich enough to provide an efficient re-identification while being sufficiently robust to remain significant through the different phenomena which may alter the appearance of a person in a video. We propose in this paper a method that encodes people's appearance through a string of salient points. The similarity between two such strings is encoded by a kernel. This last kernel is combined with a tracking algorithm in order to associate a set of strings to each person and to measure similarities between persons entering into the scene and persons who left it.
\end{abstract}

Keywords: Re-identification, String kernel, Visual surveillance.

\section{Introduction}

The purpose of re-identification is to identify people coming back into the field of view of a camera. Several types of features including interest point $[92$, histograms [310168], shape [6] and graph based representations [1711132], have been proposed in the literature. However, some features like histograms do not encode any spatial information while some others like interest point and graph based representations may induce a matching step that requires important execution times. Moreover complex features like bags or graphs [13] of interest points, RAG [2] may be sensitive to the evolution of the appearance of a person in a video due to his displacements or occlusions.

Independently of the type of features used to perform the re-identification step, re-identification methods may be split into two categories: methods of the

P. Fränti et al. (Eds.): S+SSPR 2014, LNCS 8621, pp. 333-342, 2014.

(C) Springer-Verlag Berlin Heidelberg 2014 
first group [9] compute a unique signature for each object and perform the reidentification based on this single signature. Methods of the second group 318 delay the re-identification that is then performed on a set of signatures. Using a method of the latter category imposes to base the comparison between two objects on a comparison of two sets of signatures rather than between two single signatures. However such an approach can potentially better capture the variability of the appearance of a person over a video.

Our approach belongs to the second category and describes the appearance of a person by a set of RGB string descriptors (Section 2) computed over a sliding window. A kernel between two sets of strings (Section 3) is then applied in order to encode the similarity between two persons. The integration of this kernel into a tracking method is described in Section 4 while Section 5 reports several experiments that demonstrate the validity of our approach.

\section{RGB String Descriptor Construction Scheme}

One of the main challenge in people re-identification is to capture peoples' appearance properties. As mentioned in Section 11. several modelings have been developed. However, although complex models such as graph based representation offer the advantage of a precise modeling of an object, they usually require a complex matching step and important execution times. An alternative solution consists in using a string descriptor. Indeed, a string allows an effective comparison while preserving useful information of the region of interest. Although a string usually encodes less information than a graph, we expect a greater stability of this simpler structure over time.

The first step of our method consists in separating subjects from the background. To that end, we use binary object masks [2] defined by a foreground detection with shadow removals. Each moving person within a frame is thus associated to a mask and to a bounding box characterized using a salient string. Each character of this last string is defined by a couple of coordinates (x,y) and the associated RGB image's color. The construction of a salient string is outlined in Figure 1. This construction consists of the following 3 steps:

First, we apply a Deriche edge detector on each moving person according to its binary mask within a frame.

Then, for the second stage we build a discriminating curve of the object using contour points provided by the Deriche detector. Let us consider the bounding box $W \times H$ of an object $o b j_{a}$ whose top-left corner's coordinates are denoted $\left(t l_{x}, t l_{y}\right)$. Thanks to the Deriche filter, $o b j_{a}$, should be delineated by two main contours (Figure 1 1 step-1). For each value $h \in\{0, \ldots, H-1\}$, we consider the horizontal line segment defined as the intersection between the bounding box of $o b j_{a}$ and the line $y_{h}=h+t l_{y}$. The $\mathrm{x}$ coordinate of the central point of $o b j_{a}$ at height $y_{h}$ is denoted $\bar{x}_{h}$ and is defined as the $\mathrm{x}$ coordinate of the weighted mean of all points along the line segment. More precisely, $\bar{x}_{h}$ is defined as:

$$
\forall h \in\{0, \ldots, H-1\} \quad \bar{x}_{h}=\frac{\sum_{w=0}^{W}\left|\nabla I\left(x_{w}, y_{h}\right)\right|^{2} \cdot x_{w}}{\sum_{w=0}^{W}\left|\nabla I\left(x_{w}, y_{h}\right)\right|^{2}},
$$




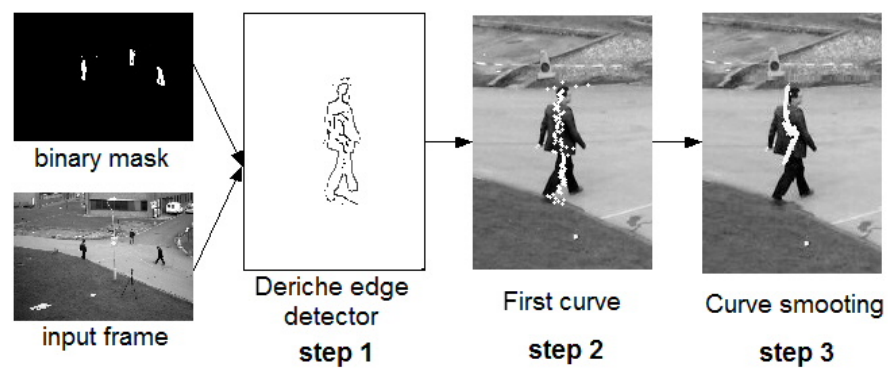

Fig. 1. RGB string construction steps

where $x_{w}=t l_{x}+w, I\left(x_{w}, y_{h}\right)$ denotes the pixel's value of $\left(x_{w}, y_{h}\right)$ and $\left|\nabla I\left(x_{w}, y_{h}\right)\right|$ is the amplitude of its gradient.

The last and third step, enforces the quality of the resulting curve. Indeed, our resulting curve (Figure 1-step-2) is sensible to small perturbations of the gradient on each line and contains important discontinuities. This last point may alter the similarity of two curves of a same person taken on two different frames. We thus propose to regularize this curve through an energy minimization framework. Our energy function (equation 21) combines two terms: the former encodes the attachment to the initial curve $\left(\bar{x}_{h}, y_{h}\right)$. The latter is a regularization term, which enforces the continuity of the curve. Hence, we assume that, the energy functional of the curve $c=\left(x_{h}^{*}, y_{h}\right)_{h \in\{0, \ldots, H-1\}}$ is defined as follows:

$$
\jmath(c)=\sum_{h=1}^{H}\left(\bar{x}_{h}-x_{h}^{*}\right)^{2}+\lambda\left(x_{h}^{*}-x_{h-1}^{*}\right)^{2},
$$

where $\lambda$ is a tuning parameter. The average coordinate $\bar{x}_{h}$ is given by equation 1 and $x_{h}^{*}$ is the corresponding final coordinate.

Minimization of equation 2 leads to search for the zeros of its gradient:

$$
\frac{\partial \jmath}{\partial x_{h}^{*}}=2(1+2 \lambda) x_{h}^{*}-2 \lambda\left(x_{h-1}^{*}+x_{h+1}^{*}\right)-2 \bar{x}_{h}=0 .
$$

Equation 3 corresponds to the formulation of a tridiagonal system which can be solved in $\mathcal{O}(n)$. This last minimization step obtained using equation 3 provides the final curve $c=\left(x_{h}^{*}, y_{h}\right)_{h \in\{0, \ldots, H-1\}}$, where each point is associated to its RGB value (Figure 1 1 step-3).

\section{People Description}

Curves encoding of people's appearance may be altered by the addition of erroneous extremities; encoding, for example, a part of the floor or a difference of sampling due to the variations of the distance between a person and the camera. In order to cope with such variations we consider each curve as a string 
and encode the similarity between two strings using the global alignment kernel defined by 4]:

$$
K_{G A}\left(s_{1}, s_{2}\right)=\sum_{\pi \in A(n, m)} e^{-D_{s_{1}, s_{2}}(\pi)},
$$

where $n$ and $m$, denote the length of the first string $s_{1}$ and the second string $s_{2}$ respectively. An alignment is noted $\pi$ and $A(n, m)$ represents the set of all alignments between $s_{1}$ and $s_{2}$. The symbol $D$ denotes the Dynamic Time Warping distance. It measures the discrepancy between two strings $s_{1}$ and $s_{2}$ according to an alignment $\pi$. Function $D$ is defined [4] as:

$$
D_{s_{1}, s_{2}}(\pi)=\sum_{i=1}^{|\pi|} \varphi\left(x_{\pi_{1}(i)}, y_{\pi_{2}(i)}\right)
$$

where $s_{1}=\left(x_{i}\right)_{i \in\{1, . ., n\}}, s_{2}=\left(y_{i}\right)_{i \in\{1, \ldots m\}}$ and function $\varphi$ corresponds to a distance function defined [4] as follows:

$$
\varphi(x, y)=\frac{1}{2 \sigma^{2}}\|x-y\|^{2}+\log \left(2-e^{-\frac{\|x-y\|^{2}}{2 \sigma^{2}}}\right),
$$

where $\mathrm{x}$ and $\mathrm{y}$ denote the RGB values of the first object and the second object respectively. Symbol $\sigma$ denotes a tuning parameter. The log term is added to the squared Euclidean distance $\|x-y\|^{2}$ in order to ensure the definite positiveness of $K_{G A}$ (equation 4) [4. Note that, using equation 5, equation 4 may be computed using a slightly modified version of the classical string edit distance algorithm. The computational complexity of equation 4 is thus bounded by $\mathcal{O}(n m)$ where $n$ and $m$ denote respectively the length of $s_{1}$ and $s_{2}$.

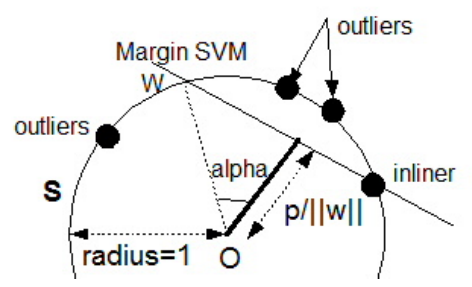

Fig. 2. Geometrical interpretation of equation 7

\subsection{People's Kernel}

As the appearance of a person evolves in a scene, due to slight changes of the pose, the use of a single string is inappropriate to identify a person. Assuming that, the appearance of a person is established in a set of successive frames, we thus describe each person by a set of salient strings. The temporal window over which this set is built is called the history tracking window (HTW). 
Each person in the video is hence not defined by a single string but by a set of strings (on HTW). This set may include outlier strings, due to slight changes of the pose or occlusion. The construction of a representative string based on a simple average of all the strings of a set (in the Hilbert space defined by the kernel) may be sensible to such outliers. We thus suggest to enforce the robustness of our representative string through the use of an one class SVM classifier.

Let $\mathcal{H}$ denotes the Hilbert space defined by $K_{G A}$ (equation 4). In order to get a robust model encoding the mean appearance of a person, we first use $K_{G A}$ to project the mapping of all strings onto the unit-sphere of $\mathcal{H}$. This operation is performed by normalizing our kernel [5]. Following [5], we then apply a one class $\nu$-SVM on each set of strings describing a person. From a geometrical point of view, this operation is equivalent to model the set of projected strings by a spherical cap defined by a weight vector $w$ and an offset $\rho$ both provided by the $\nu$ SVM algorithm. These two parameters define the hyper plane whose intersection with the unit sphere defines the spherical cap. Strings whose projection on the unit sphere lies outside the spherical cap are considered as outliers. Each person is thus encoded by a triplet $(w, \rho, S)$ where $S$ corresponds to the set of strings and $(w, \rho)$ are defined from a one class $\nu$-SVM. Figure 2 gives the geometric interpretation of $(w, \rho)$; The parameter $w$ indicates the center of the spherical cap and may be intuitively understood as the vector encoding the mean appearance of a person over its HTW window. The parameter $\rho$ influence the radius of the spherical cap and may be understood as the extend of the set of representative strings in $S$.

Let $P_{A}=\left(w_{A}, \rho_{A}, S_{A}\right)$ and $P_{B}=\left(w_{B}, \rho_{B}, S_{B}\right)$ denote two triplets encoding two persons $A$ and $B$. The distance between $A$ and $B$ is defined from the angle between vectors $w_{A}$ and $w_{B}$ defined as follows [5]:

$$
d_{\text {sphere }}\left(w_{A}, w_{B}\right)=\arccos \left(\frac{w_{A}^{T} K_{A, B} w_{B}}{\left\|w_{A}\right\|\left\|w_{B}\right\|}\right)
$$

where $\left\|w_{A}\right\|$ and $\left\|w_{B}\right\|$ denote the norms of $w_{A}$ and $w_{B}$ in $\mathcal{H}$ and $K_{A, B}$ is a $\left|S_{A}\right| \times\left|S_{B}\right|$ matrix defined by $K_{A, B}=\left(K_{\text {norm }}\left(t, t^{\prime}\right)\right)_{\left(t, t^{\prime}\right) \in S_{A} \times S_{B}}$, where $K_{\text {norm }}$ denotes our normalized kernel.

Based on $d_{\text {sphere, }}$ the kernel between $A$ and $B$ is defined as the following product of RBF kernels:

$$
K_{\text {change }}\left(P_{A}, P_{B}\right)=e^{\frac{-d_{\text {sphere }}^{2}\left(w_{A}, w_{B}\right)}{2 \sigma_{\text {moy }}^{2}}} e^{\frac{-\left(\rho_{A}-\rho_{B}\right)^{2}}{2 \sigma_{\text {origin }}^{2}}},
$$

where $\sigma_{m o y}$ and $\sigma_{\text {origin }}$ are tuning variables.

\section{Re-identification}

Our tracking algorithm is based on a previous work 13. The tracking algorithm uses four labels 'new', 'get-out', 'unknown' and 'get-back' with the following 


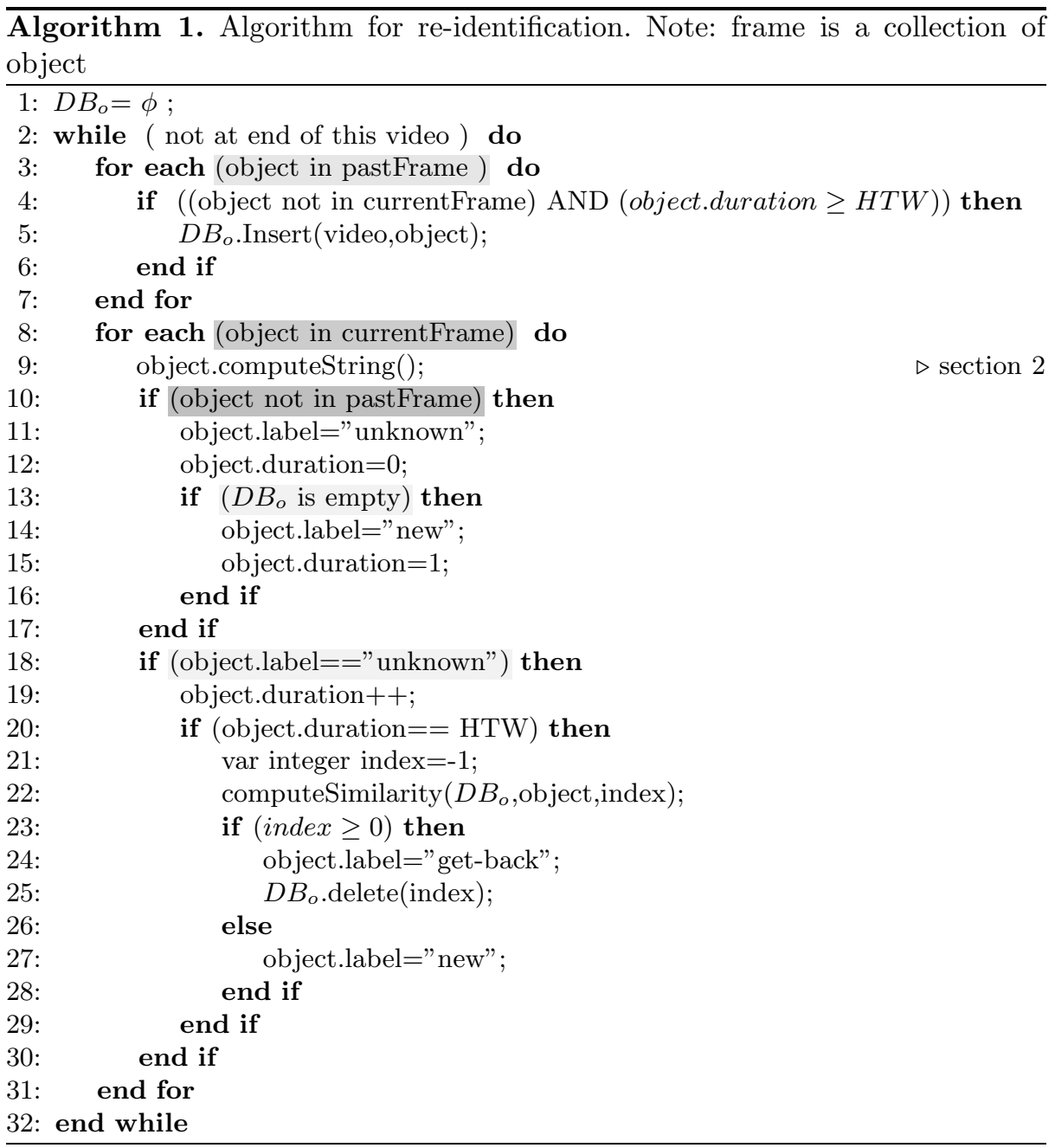

meaning: 'new' refers to an object classified as new, 'get-out' represents an object leaving the scene, 'unknown' describes a query object (an object recently appeared, not yet classified) and 'get-back' refers to an object classified as an old one after a re-identification step. All masks detected in the first frame of a video are considered as new persons. The proposed re-identification approach is depicted in Algorithm 1 .

- 'get-out' processing (lines 3 to 7): when an object leaves the scene its triplet $P=(w, \rho, S)$ (Section 3) computed over the last $|H T W|$ frames is stored in an output object data base noted $D B_{o}$.

- 'new' processing (lines 10 to 17): when an 'unknown' person is found and $D B_{o}$ is empty we label this 'unknown' person as new. 
- 'unknown' processing (lines 18 to 30) when an 'unknown' person is found and $D B_{o}$ is not empty we should postpone the identification of this 'unknown' person; The 'unknown' person is tracked on $|H T W|$ frames in order to have its description by a triplet $(w, \rho, S)$. Using this description we calculate the value of kernel $K_{\text {change }}$ (equation 8 ) between this 'unknown' person and all 'get-out' persons present in our database (line 22). Similarities between the 'unknown' person and the 'get-out' persons are sorted in decreasing order so that the first 'get-out' person of this list corresponds to the best candidate for a re-identification. Our criterion to map an 'unknown' person to 'getout', and thus to label it as 'get-back' is based on a threshold on the first two maximal similarity values $\max _{k e r}$ and $\max _{2}$ of the list of similarities $\left(\max _{2} \leq \max _{K e r}\right)$. This criterion called, SC is defined as $\max _{k e r}>t h_{1}$ and $\frac{\max _{2}}{\text { max }_{\mathrm{K} e r}}<t h_{2}$, where $t h_{1}$ and $t h_{2}$ are experimentally fixed thresholds.

Classically, any tracking algorithm has to cope with many phenomena such as occlusions. In this paper we limit the study to overlapping bounding boxes. When an overlap greater than an experimentally fixed threshold occurs between two bounding boxes, an occlusion is found. We assume two kinds of occlusions: partial occlusions; where the occluded object remains visible and severe occlusions; where the occluded object is completely hidden. If two or more objects (detected at time $t$ ) merge together (at time $t+1$ ) to form one new object, this object is deemed to be a group rather than an occlusion. Group cases are not considered in this work.

\section{Experiments}

The proposed algorithm has been tested on v01 and v05 video sequences of the PETS'09 S2L1 dataset1. Each sequence contains multiple persons and occlusion cases. We have to notice that all the tuning parameters used in this work are set by cross-validation. As the used dataset does not contains a training set, the tuning parameters are set using the test dataset.

In our first experiment we have evaluated how different values of the length of HTW affect the re-identification accuracy. Figure 3 shows the effects for HTW changes on the true positive measurement for each view. The obtained results show that, v01 performs at peak efficiency for $\mathrm{HTW}=30$. Video v05 reach its optimum at HTW=20. These curves also show that the length of HTW is not a crucial parameter of our method.

In a second experiment we show the improvement of the proposed kernel with respect to a histogram based approach. Similarly to [15] where histograms are defined from the already extracted blob (segmented parts), we propose the following histograms construction scheme: color histograms are computed on HTW frames for both the query object and each 'get-out' persons contained in $D B_{o}$. Then, we try to map the query object with one of the 'get-out' objects already stored in $D B_{o}$ using EMD distance [14] between histograms. If a map is found,

\footnotetext{
${ }^{1}$ Available at http://www.cvg.rdg.ac.uk/PETS2009/a.html
} 


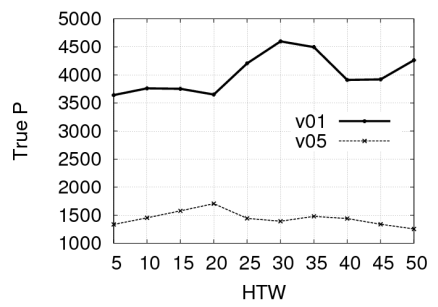

Fig. 3. HTW effects

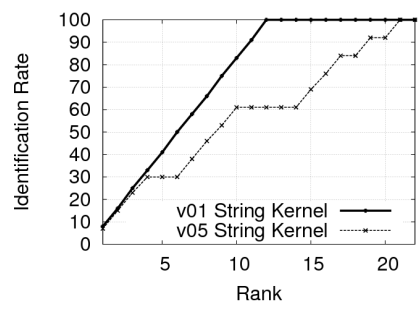

Fig. 4. CMC curves

the query object gets the label of the mapped 'get-out' object, and we update $D B_{o}$. Otherwise, we create a new label for the query object. The map criterion used here is similar to the above SC criterion (Section 4), nevertheless the best candidate corresponds to the minimum since we use distances. Table 1 reports the comparison results between histogram-based, kernel-based approaches and graph approach of [2]. As it can be seen from Table 1, the performance of the proposed kernel is superior to the histogram method as expected. Furthermore, the results of the proposed kernel give lower values than the graph approach regarding v05, while the results are clearly better for v01. We attribute this to the high detection accuracy in v01. Furthermore, v05 contains a lot of severe occlusions that are not specifically addressed in the proposed method. Indeed on such severe occlusions a large part of a person is usually hidden by another person.

To validate our method of re-identification we used the Cumulative Matching Characteristic (CMC) curves. The CMC curve represents the percentage of times the correct identity match is found in the first $n$ matches. Figure 4 shows the CMC curves for the two views. We can see that the performance of v01 is much better than that of v05. This last result being due to the large number of occlusions occurring in v05.

In a third experiment, we assess the statistical performance of the proposed kernel. To this end, we iteratively shift the beginning of the video by a unit of 50 frames to obtain 15 subsets of the original video i.e., $t_{0} \in[50,750]$. We thus reported for each set the TrueP and the FalseP values. Finally we computed the mean $(a v g)$ and the standard deviation $(\sigma)$ of the obtained results. As expected, the results are coherent with Table1 (line 2) since the resulting couples $(a v g, \sigma)$ regarding TrueP are equal to $(0.90,0.08)$ and $(0.66,0.165)$ for v01 and v05 respectively.

Table 1. Comparison results

\begin{tabular}{|c|c|c|c|c|}
\hline \multirow{2}{*}{} & \multicolumn{2}{|c|}{ view01 } & \multicolumn{2}{c|}{ view05 } \\
\cline { 2 - 5 } & TrueP & FalseP & TrueP & FalseP \\
\hline histogram & $51.46 \%$ & $48.54 \%$ & $49.91 \%$ & $50.09 \%$ \\
\hline kernel & $100 \%$ & $0 \%$ & $62.61 \%$ & $33.39 \%$ \\
\hline graph of [2] & $81 \%$ & $7 \%$ & $83 \%$ & $13 \%$ \\
\hline
\end{tabular}

Table 2. Evaluation results

\begin{tabular}{|c|c||c|c|c|}
\hline \multirow{2}{*}{ View } & work of [1] & \multicolumn{3}{c|}{ current work } \\
\cline { 2 - 5 } & MODA & MODA & MOTA & SFDA \\
\hline v01 & 0.67 & 0.97 & 0.97 & 0.91 \\
\hline v05 & 0.72 & 0.60 & 0.60 & 0.81 \\
\hline
\end{tabular}


We also used the exhaustive comparison of 13 methods defined in 7 in order to compare our results to the state of the art. The study [7] did a quantitative evaluation of the results submitted by contributing authors of the two PETS workshops in 2009 on PETS'09 S2.L1 dataset. We noticed that the submitted results of [1] outmatch all other methods using the MODA, MOTA, MODP, MOTP SODA and SFDA metrics described in [12]. Therefore, we only compare our results to that last method. Table 2 depicts the following: the left column shows the best results [1] obtained by methods described in [7] on each video. The second column of Table 2 shows that our method obtains a lower MODA index than [1] on v05 but clearly outperform this last method on v01. These results may again be explained by the high number of occlusions in v05 which are overcomed by [1] using multiple views of each person while the present method is restricted to a single view. These results indicate thus the relevance of the proposed re-identification method when objects are not severely occluded.

\section{Conclusion}

In this work, we addressed the people re-identification problem by proposing a new approach based on RGB string kernels. A benchmark public dataset was used to validate our method. Our results show that the proposed approach outperforms state-of-the art methods when few severe occlusions occur.

Our future research will focus on the investigation of occlusion and group problems still using a single camera. To handle these phenomena, we should for each object severely occluded or entering into a group, suspend the update of its curves for the frames where it is hidden. Indeed in such cases no reliable feature may be extracted to characterize hidden persons.

\section{References}

1. Berclaz, J., Shahrokni, A., Fleuret, F., Ferryman, J., Fua, P.: Evaluation of probabilistic occupancy map people detection for surveillance systems. In: Proceedings of the IEEE International Workshop on Performance Evaluation of Tracking and Surveillance (PETS), pp. 55-62 (2009)

2. Brun, L., Conte, D., Foggia, P., Vento, M.: People re-identification by graph kernels methods. In: Jiang, X., Ferrer, M., Torsello, A. (eds.) GbRPR 2011. LNCS, vol. 6658, pp. 285-294. Springer, Heidelberg (2011)

3. Cong, D.N.T., Khoudour, L., Achard, C., Meurie, C., Lezoray, O.: People re-identification by spectral classification of silhouettes. Signal Processing 90(8), 2362-2374 (2010)

4. Cuturi, M.: Fast global alignment kernels. In: Getoor, L., Scheffer, T. (eds.) ICML, pp. 929-936. Omnipress (2011)

5. Desobry, F., Davy, M., Doncarli, C.: An online kernel change detection algorithm. IEEE Transactions on Signal Processing 53(8-2), 2961-2974 (2005)

6. Doretto, G., Sebastian, T., Tu, P., Rittscher, J.: Appearance-based person reidentification in camera networks: problem overview and current approaches. Journal of Ambient Intelligence and Humanized Computing 2, 127-151 (2011) 
7. Ellis, A., Shahrokni, A., Ferryman, J.M.: Pets2009 and winter-pets 2009 results: a combined evaluation. In: 2009 Twelfth IEEE International Workshop on Performance Evaluation of Tracking and Surveillance, Snowbird, USA, Conference held December 7-9. IEEE (2009)

8. Farenzena, M., Bazzani, L., Perina, A., Murino, V., Cristani, M.: Person reidentification by symmetry-driven accumulation of local features. In: Proceedings of the 2010 IEEE Computer Society Conference on Computer Vision and Pattern Recognition (CVPR 2010). IEEE Computer Society, San Francisco (2010)

9. Hamdoun, O., Moutarde, F., Stanciulescu, B., Steux, B.: Person re-identification in multi-camera system by signature based on interest point descriptors collected on short video sequences. In: ICDSC, pp. 1-6. IEEE (2008)

10. Ijiri, Y., Lao, S., Han, T.X., Murase, H.: Human re-identification through distance metric learning based on jensen-shannon kernel. In: VISAPP (1), pp. 603-612 (2012)

11. Iodice, S., Petrosino, A.: Person re-identification based on enriched symmetry salient features and graph matching. In: Carrasco-Ochoa, J.A., Martínez-Trinidad, J.F., Rodríguez, J.S., di Baja, G.S. (eds.) MCPR 2012. LNCS, vol. 7914, pp. 155164. Springer, Heidelberg (2013)

12. Kasturi, R., Goldgof, D., Soundararajan, P., Manohar, V., Garofolo, J., Bowers, R., Boonstra, M., Korzhova, V., Zhang, J.: Framework for performance evaluation of face, text, and vehicle detection and tracking in video: Data, metrics, and protocol. Pattern Analysis and Machine Intelligence 31(2), 319-336 (2009)

13. Mahboubi, A., Brun, L., Conte, D., Foggia, P., Vento, M.: Tracking system with re-identification using a graph kernels approach. In: Wilson, R., Hancock, E., Bors, A., Smith, W. (eds.) CAIP 2013, Part I. LNCS, vol. 8047, pp. 401-408. Springer, Heidelberg (2013)

14. Rubner, Y., Tomasi, C., Guibas, L.J.: The earth mover's distance as a metric for image retrieval. International Journal of Computer Vision 40(2), 99-122 (2000)

15. Satta, R., Fumera, G., Roli, F., Cristani, M., Murino, V.: A multiple component matching framework for person re-identification. In: Maino, G., Foresti, G.L. (eds.) ICIAP 2011, Part II. LNCS, vol. 6979, pp. 140-149. Springer, Heidelberg (2011)

16. Schwartz, W.R., Davis, L.S.: Learning Discriminative Appearance-Based Models Using Partial Least Squares. In: Brazilian Symposium on Computer Graphics and Image Processing, pp. 322-329 (2009)

17. Wang, X., Doretto, G., Sebastian, T., Rittscher, J., Tu, P.H.: Shape and appearance context modeling. In: ICCV, pp. 1-8. IEEE (2007)

18. Zhao, S., Precioso, F., Cord, M.: Spatio-temporal tube data representation and kernel design for svm-based video object retrieval system. Multimedia Tools Appl. 55(1), 105-125 (2011) 